\title{
GENERATION OF THREE-DIMENSIONAL FREE-STANDING MICRO-OBJECTS BY LASER CHEMICAL PROCESSING
}

\author{
O. LEHMANN and M. STUKE \\ Max-Planck-Institut für Biophys.Chemie, Postfach 2841. \\ D-3400 Göttingen, Germany
}

\begin{abstract}
Three-Dimensional free standing micro-objects in the micrometer range were created using Laser Chemical Vapor Deposition (LCVD) of aluminum from aluminum-trihydridetrimethylamine. A dense grid of thin lines (about $10 \mu \mathrm{m}$ diameter) was deposited on a preformed polycarbonate substrate using a two-step-process. The substrate was removed by dissolving it in a suitable solvent after ablating an unsoluble by-product by UV excimer laser pulses. The result is a free-standing three-dimensional aluminum grid micro-structure.
\end{abstract}

\section{Introduction}

Surfaces can be processed chemically when they are in contact to a potentially chemically reactive system and/or exposed to beams of energetic radiation such as charged (ions, electrons) or neutral particles or photons. Photons offer the advantage that they can penetrate high density environments on the order of $10^{23} \mathrm{~cm}^{-3}$ without distortion and uncontrolled attenuation of the beam. With lasers as photon sources, the energetic irradiation can be well controlled to dimensions smaller than $1 \mu \mathrm{m}$ and to ultrashort exposure times well below $10^{-12} \mathrm{~s}$, if useful or necessary. Spatial control of the surface irradiation is easily achieved, either by scanning the laser focus position ("laser-direct-write") over the surface, by patterned irradiation through a contact mask, or imaged to the surface to be processed. Typical examples are deposition of materials by Laser Chemical Vapor Deposition ("LCVD") [1], Laser Activated Deposition ("LAD") [2] using two-step processes, and laser induced material removal such as etching and ablation .

Structured deposition of various materials is of considerable interest to many fields of science and technology. For (micro-) electronics, laser deposition of metals, semiconductors, passivation layers and superconductors has to be achieved in a way compatible with other processing steps involved in microelectronics fabrication. 
Up to now, laser induced deposition has - with a few exceptions [3] - mainly been applied to planar substrates.

In the following, the generation of three-dimensional free-standing micro-objects by laser chemical processing will be described using a combination of LCVD with a continuous wave (cw) $\mathrm{Ar}^{+}$-laser and pulsed UV excimer laser ablation. To our knowledge, this is the first example realized.

\section{Experimental}

Using pre-formed and soluble three-dimensional substrates of various materials, free standing micro-objects are generated by Laser Chemical Vapor Deposition (LCVD). These substrates are prepared by shaping their surfaces in a way, that the topology of the substrate to be written on determines the resulting shape of the generated 3-D object. For this purpose, all locations of the substrate surface must be accessible by the laser beam. Only for those few concave forms that are not directly accessible with this technique, step-wise formation of the topology may be required.

Pre-forming can be done by conventional methods such as cutting or etching as well as by laser ablation. The example mentioned in this paper was produced by conventional milling.

Since the substrate surface characteristics strongly determine the quality and homogeneity of the laser-deposited metal structure, smooth surfaces are required and sharp edges should be avoided, if possible. In order to ease handling of the finished 3-D object, the soluble substrate is connected with one side to a base object consisting of a material not affected by the solvent used to remove the substrate after laser processing.

As a first example, aluminum is deposited from the adduct precursor aluminum-trihydride-trimethylamine, $\left(\mathrm{CH}_{3}\right)_{3} \mathrm{~N} \cdot \mathrm{AlH}_{3} \cdot \mathrm{N}\left(\mathrm{CH}_{3}\right)_{3}$ onto polycarbonate. Because of the low melting point of polycarbonate $\left(120^{\circ} \mathrm{C}\right)$, a precursor with a low decomposition threshold is required, so that possible damage to the substrate is reduced to a minimum. The precursor chosen $[4,5,6,7]$ is an ideal compound from this viewpoint. Moreover, the purity and quality of the deposited aluminum is good compared to other precursors.

A continuous $\mathrm{Ar}^{+}$- $\operatorname{laser}(\lambda=514.5 \mathrm{~nm})$ is used as photon source. The laser beam is coupled into the beam path of a microscope objective and - passing a quartz window of $2 \mathrm{~mm}$ thickness - is focused to the substrate surface [8] with a focus diameter on the order of $1 \mu \mathrm{m}$ [9]. The focal 
planes of both the laser beam and the view through the microscope are aligned so that the laserdirect-writing process can be viewed directly.

A schematic view of the experimental setup is shown in Fig. 1.

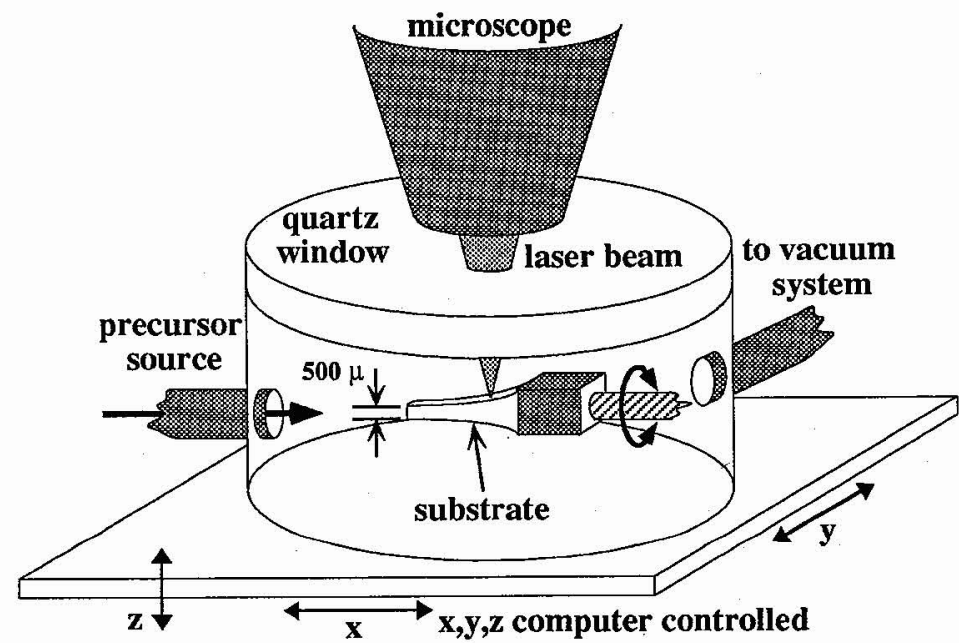

Fig. 1: Schematic view of the experimental setup. The substrate can be moved under computer control in all room directions (xyz) and turned around one axis. The laser beam is focused to the surface of a pre-formed substrate. A dense grid with a diameter around $10 \mu \mathrm{m}$ of aluminum is deposited. This grid forms the finished 3-D structure defined by the shape of the 3-D substrate. After dissolving the substrate, a self-supporting 3-D structure remains.

Using a computerized scanning table, arbitrary lines of aluminum are deposited. The characteristics of the deposited lines depend on various parameters, such as laser scan speed, precursor and total pressure, laser power, focus, substrate surface quality and gas purity. All these factors have to be optimized to create high quality, small diameter lines in the $\mu \mathrm{m}$ range. To avoid melting of the substrate and to get rigid lines with a circular cross-section, a two-step process is used: During the first step, a thin line is created with relatively high scan speed (200$300 \mu \mathrm{m} / \mathrm{s}$ ) and low laser output power (20-30 mW) under microcomputer control. Afterwards, this line is amplified with somewhat lower scan speed (ca. $150 \mu \mathrm{m} / \mathrm{s}$ ) and higher laser power (up to $60 \mathrm{~mW}$ ). The major advantage compared to a one-step deposition with comparable line size is, that the aluminum line created by the first step absorbs much of the incoming energy when performing the second step. Because of the higher thermal conductivity of aluminum compared to polycarbonate, the temperature gradient in the focus area is reduced considerably. In spite of higher laser intensity, the focus temperature is quite low, and the increase in laser 
power does not lead to local melting of the substrate, but faster line growth. This process is applied to all lines on each side of the substrate so that it is coated by a software controlled structure, in this case a dense grid of aluminum lines with an average line spacing of about 150 $\mu \mathrm{m}$. The grid is connected by LCVD to the base object mentioned earlier.

The deposition process leaves a thin layer of an unsoluble, not yet characterized by-product on the surface of the substrate. This obviously polymeric by-product would delay the solution process described later, and its existence on the finished object is not desired. Therefore, it is removed by laser ablation using unfocused UV excimer laser radiation $(\lambda=248 \mathrm{~nm})$. After typically five laser pulses, the by-product layer is ablated completely, whereas the aluminum grid and the polycarbonate substrate remain intact, as required.

The compound structure is then dissolved in liquid chloroform $\left(\mathrm{CH}_{3} \mathrm{Cl}\right)$ at room temperature. This procedure removes the polycarbonate substrate, only used to define the three-dimensional shape, and the aluminum grid structure remains unaffected. During the solution process, attention must be paid in order not to destroy the sensitive grid structure created. For example, the surface tension of the solvent can be strong enough to break up lines and destroy the structure if not dissolved properly. Other techniques for substrate removal may be used if necessary.

\section{Result}

The generated 3-D object consists of a free standing aluminum grid structure (base length 3000 $\mu \mathrm{m}$, height $7000 \mu \mathrm{m}$ for this example) with a grid diameter around $10 \mu \mathrm{m}$, depending on deposition and laser conditions. A typical example is shown in Fig. 2. It can be seen on the photo that virtually all parts of the grid are intact. This demonstrates for the first time that it is possible to create self-supporting three-dimensional objects using the depicted combination of deposition and ablation laser techniques.

Because of the additional use of the base object mentioned earlier in this paper, the finished object can be handled very easy by touching only this base object. Without this object, almost every try to snatch the sensitive grid would lead to destruction of the structure. 


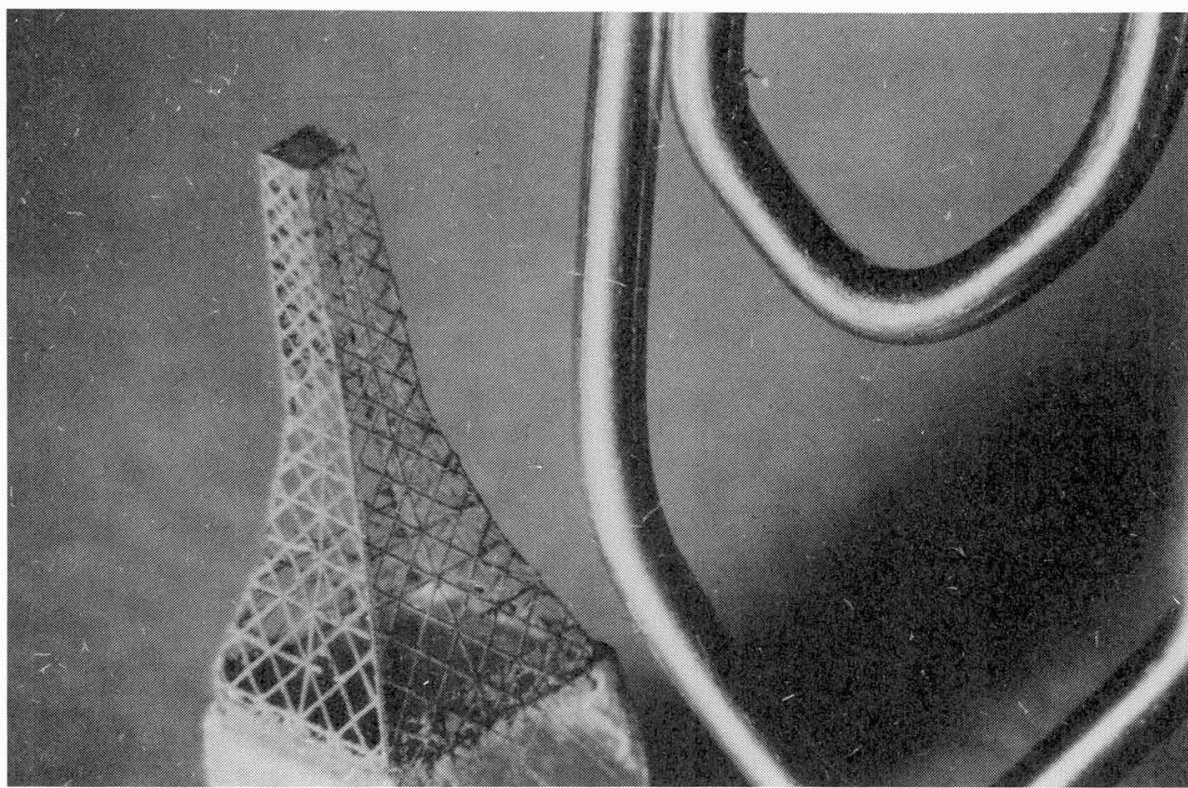

Fig. 2: Total view of the finished three-dimensional aluminum grid structure. For the ease of handling, the structure is connected to the base object at the bottom of the photo by laser CVD.

\section{Summary}

Three-dimensional free standing micro-objects consisting of an aluminum grid in the micrometer range were created using Laser Chemical Vapor Deposition (LCVD) of aluminum from aluminum-trihydride-trimethylamine on pre-formed soluble polycarbonate using a continuous wave $\mathrm{Ar}^{+}$-laser with $\lambda=514.5 \mathrm{~nm}$. The aluminum is deposited in a thin line form (diameter about $10 \mu \mathrm{m}$ ) that defines a dense grid (line spacing about $150 \mu \mathrm{m}$ ) using a two-step process. The polycarbonate substrate is removed by solution in chloroform after ablating an unsoluble by-product on the surface by pulsed UV excimer laser radiation. The resulting selfsupporting three-dimensional aluminum structure is connected to a metal base to ease handling.

Acknowledgements: We would like to acknowledge help by H. W. Roesky, K. Müller and O. Gottsleben. Support by F. P. Schäfer and financial support by BMFT 13 N 5398/7 and $13 \mathrm{~N}$ $5622 / 6$ are gratefully acknowledged.

\section{References:}

1 D. Bäuerle, "Chemical Processing with Lasers", Springer Verlag, Heidelberg 1986.

2 A. Bauer, J. Ganz, K. Hesse, E. Köhler, Applied Surface Science 46 (1990) $113 f f$.

3 A. F. Bernhardt in "Surface Processing and Laser Assisted Chemistry", ed. I.W. Boyd, E. Fogarassy, M. Stuke 


\footnotetext{
${ }^{4}$ K. Ziegler, W.-R. Kroll, W. Larbig, O.-W. Steudel, Ann. Chem. 629 (1960) 53.

5 T. H. Baum, C. E. Larson, R. L. Jackson, Appl. Phys. Lett. 55 (1989) 1264.

6 O. Gottsleben, H.W. Roesky, M. Stuke, Advanced Materials 3 (1991) 201.

${ }^{7}$ commercially available from Kali-Chemie Hannover (Dr. Döllein)

8 O. Gottsleben, M. Stuke, Appl. Phys. Lett. 52 (1988) 2230.

${ }^{9}$ P. Fischer, B. Schreiber, Jenoptik Carl Zeiss Jena GmbH, O-6900 Jena.
} 